\title{
Modeling and Analyses the Equivalent-Schema Models for OSRR and COSRR Coupled to Planar Transmission Lines by Scattering Bond Graph
}

\author{
Islem Salem ${ }^{1}$, Hichem Taghouti ${ }^{2}$, Abdelkader Mami ${ }^{3}$ \\ UR-LAPER, University of Tunis El Manar, Tunis, Tunisia \\ Tunis, Tunisia
}

\begin{abstract}
Following consumer demand, international competition has become increasingly high, suddenly industrialists are mobilizing to meet the requirements. Faced with this challenge, and in order to be able to respond to these prerequisites, manufacturers are looking for techniques that will allow them to gain productivity by increasing the rate of perfection before going to manufacturing. Modeling presents the most important phase in a construction chain since it allows not only analysis and understanding of the physical system but also to improve its behavior according to the desired objective from the design phase. The results presented in this article concern the modeling of the transmission lines of metamaterials loaded with OSRR "Open Split-Ring Resonators" and COSRR "Complementary Open Split-Ring Resonators" resonators, with the aim of improving analysis, synthesis and understanding of this system. By using the Scattering Bond Graph technique, which improves the adaptation of the impedance, and reduces the bandwidth. This technique allows us to deduce the scattering parameters (matrix [S]) of the OSRR / COSRR TL elements from the wave matrix [W], hence this matrix is determined through on the specific properties of the equivalent Bond Graph presentation based on the notion of causality.
\end{abstract}

Keywords-Scattering Bond Graph (SBG); metamaterials; wave matrix [W]; matrix scattering [S]; transmission line; OSRR and $O C S R R$

\section{INTRODUCTION}

Recently, a new expression appeared in the universe of the microwave, it is the metamatriaux [1] (artificial metallodiélecctric structuring properties not found in nature). From a historical point of view, the electromagnetic properties of these environments have been studied by Victor. G. Veslago in 1968 [2] , the term metamaterials has been synthesized by Rodger. M. Walser in 1999 [3], and the first actual realization was made by R. A. Schelby in 2001 [4], these structures are currently subjects of study in full development. These materials can improve the realization of several microwave devices and at the same time promise new types of more efficient circuits and more miniature.

Several metamatrial-based transmission line structures have been studied, these lines are implemented in charge of a transmission line with resonators such as OSRR and OCSRR [5], [6]. These structures have already contributed to the growth of development of the design of new microwave circuit systems, but they still have drawbacks.
However, the traditional techniques of studying in [1], [7] this type of structure can be long, complicated and require a thorough knowledge of the electromagnetic field, adding that they are expensive, without forgetting that it poses a huge problem of impedance adaptation. For these reasons, we propose a technique of study allowing to evolve less complicated in terms of extraction of diffusion parameters (distribution parameters), and which proved the effectiveness of the physical models of system with microwaves, as well as than its precision in terms of adaptation. This technique is called "Scattering Bond Graph".

In this paper, we study, after modeling the proposed equivalent circuits, the physical characteristics of resonators loaded with transmission lines using the Scattering Bond Graph methodology. In order to provide a general method and easier to handle for OSRR and OCSRR coupled to planar transmission lines.

\section{SCATTERING BOND GRAPH}

The conjunction between the scattering formalism [8] (method used in high frequency, electricity or electronics to describe electrical network power behavior according to network to enter) and the bond graph approach [9] (multidisciplinary physical systems modeling method) gave rise to the "Scattering Bond Graph" methodology. this methodology, which was launched in 2010 [10], has received a great deal of in the radiorfrequency microwave.

The Bond Graph is an approach to represent energy exchanges by links in terms of flow and effort between the elements of the physical system called ports. The power exchange between the two ports $\mathrm{A}$ and $\mathrm{B}$ of a system is represented by a half arrow (link indicating the direction of power) as shown in Fig. 1.

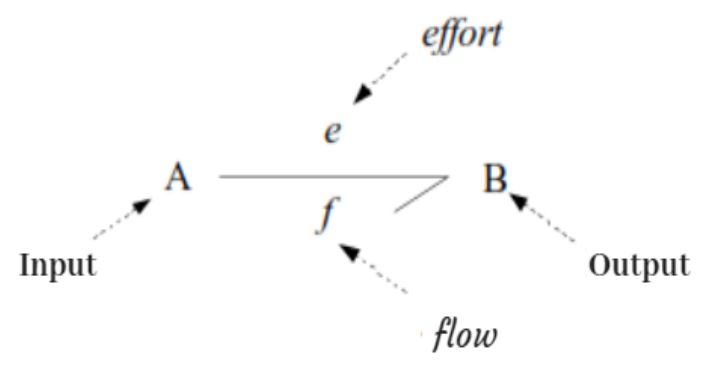

Fig. 1. Representing a Link between Two Ports. 
Causality makes it possible to show graphically the cause effect relationships and the calculation orientation of the characteristic equations [10] (the causal trait is placed on the side of the element on which the effort is imposed). There are two possible conventions for showing causality: [11].

- The first convention represented in Fig. 2 shows the flow-effort relationship between the two elements A and $\mathrm{B}$. When $\mathrm{A}$ sends an effort to $\mathrm{B}, \mathrm{B}$ responds by sending a flow to $\mathrm{A}$.

- The second convention represented in Fig. 3 shows the flow-effort relationship between the two elements A and $\mathrm{B}$. When $\mathrm{A}$ sends a flow to $\mathrm{B}, \mathrm{B}$ responds by sending an effort to A.

In the case where the efforts are equal (only one link with a causal line near the junction) we gotten the 0 -junction (Fig. 4 ), [10] and in the case where the flows are equal (only one link without causal line near the junction) we gotten 1junction (Fig. 5) [11].

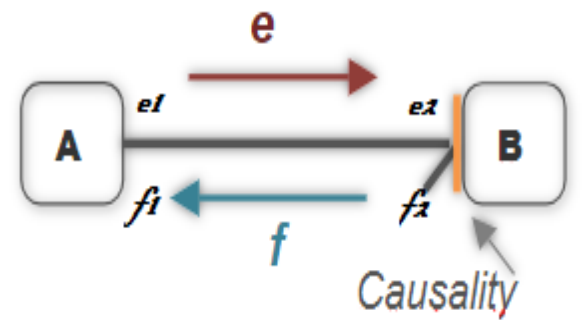

Fig. 2. A Sends an effort to B, B Responds by Sending a flow to A.

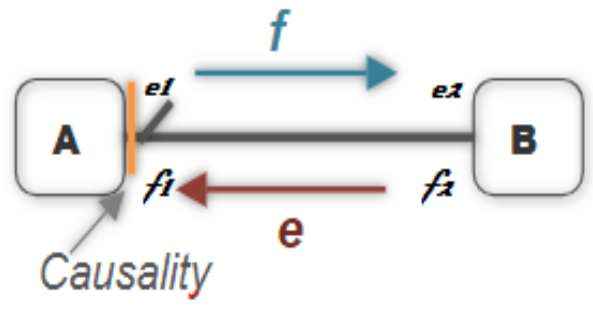

Fig. 3. A Sends a flow to B, B Responds by Sending an effort to A.

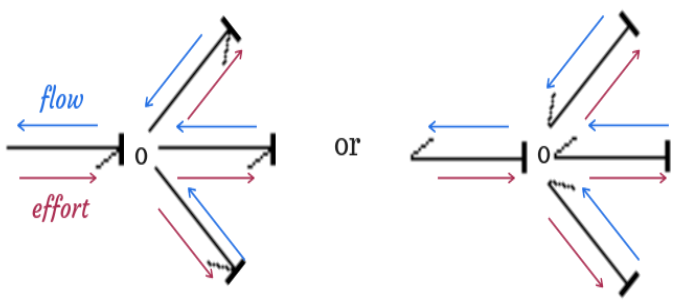

Fig. 4. Multiport 0-Junction.

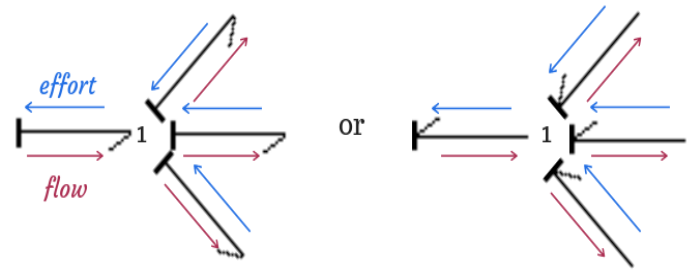

Fig. 5. Multiport 1-Junction.
Thanks to this methodology, all the physical systems can represent and interpret by lines and symbols what allows us to study them.

Indeed the Scattering Bond Graph, is a particular form that allows us to interpret, model and study the complex physical systems in microwave.

\section{MODELING AND ANALYSIS OSRR AND OCSRR LOADED TRANSMISSION LINE}

A physical system can be represented by a two-port network (a circuit with two pairs of terminals), which contains several components that interact with other circuits via ports.0.

Fig. 6 shows a two-port system where incident $\left(a_{n}\right)$ and reflected $\left(b_{n}\right)$ waves are indicated at both ports. These waves are linked by matrix relations and express the relations between the wave at the inputs and the waves at the outputs of the system.

The determination of the parameter values of the scattering matrix (called matrix [S]) [12], using the Scattering Bond Graph methodology, is done in a simpler way than the old methods, through the relation (1) which makes the connection between the wave matrix (called matrix [W] where the parameters this matrix are extracted directly from the Bond Graph model) and the matrix [S].

$[\mathrm{S}]=\left(\begin{array}{cc}\frac{W_{11}}{W_{22}} & \frac{W_{22} W_{11}-W_{12} W_{21}}{W_{22}} \\ \frac{1}{W_{22}} & \frac{-W_{21}}{W_{22}}\end{array}\right)$

In the following, the syntheses will be presented, for the purpose of implementing the graphic diffusion link methodology which uses as a new design technique which makes it possible to reduce the size of the resonators, in itself based on the particular models and characteristics of diffusion bond graph which can be simply simplified.

\section{A. Synthesis of OSRR Synthesis of OSRR (Open Spilt-Ring Resonator)}

The OSRR was introduced for the first time in [13]. OSRR is the open cell of the SRR (split ring resonator). These open cells are characterized by a negative permittivity on a welldefined frequency band. These structures can be easily excited with a normal electric field at inclusion and so the OSRRs are very easy to integrate into planar and transmission line based circuits.

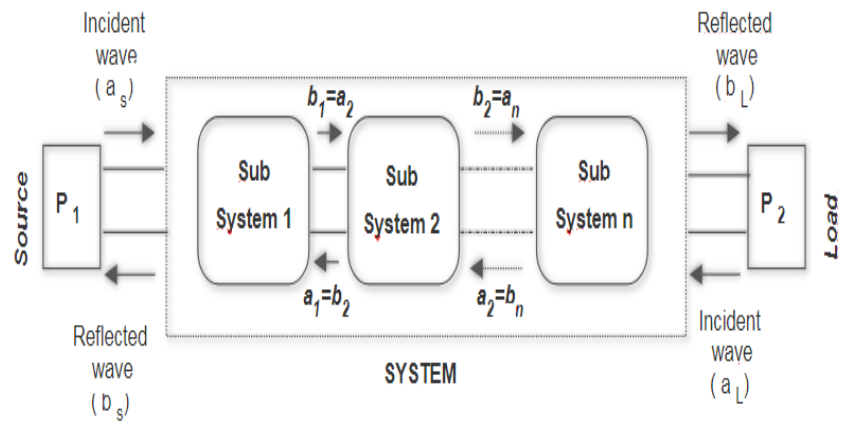

Fig. 6. Two-Port System. 
The structure of OSRR can be realized by two rings which are placed concentrically with slots placed in the same position [14].

The configuration of the OSRR is illustrated in Fig. 7 where the substrate considered is the Rogers RO3010 with a thickness $h_{\text {ocrr }}=0,254 \mathrm{~mm}$ and a dielectric constant $\varepsilon_{\mathrm{r}}=11,2$.

Fig. 8 represents the response of the simulation of scattering parameters $[\mathrm{S}]$ of the topology of a series connected OSRR in a CPW transmission line shown in Fig. 7.

The proposal of the resonator equivalent circuit OSRR can be represented by a $\pi$ circuit (presented by localized elements $\mathrm{C}_{\mathrm{s}}, \mathrm{L}_{\mathrm{s}}, \mathrm{C}_{1}$ and $\mathrm{C}_{2}$ ) as shown in Fig. 9 with a source and load equal to $50 \mathrm{ohms}$.

The acausal Bond Graph model of a resonator of the OSRR type can be represented by the Fig. 10 [11] [12].

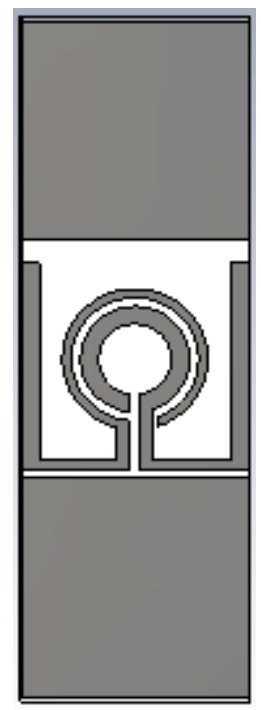

Fig. 7. Topologie of a series connected OSRR in a CPW transmission line

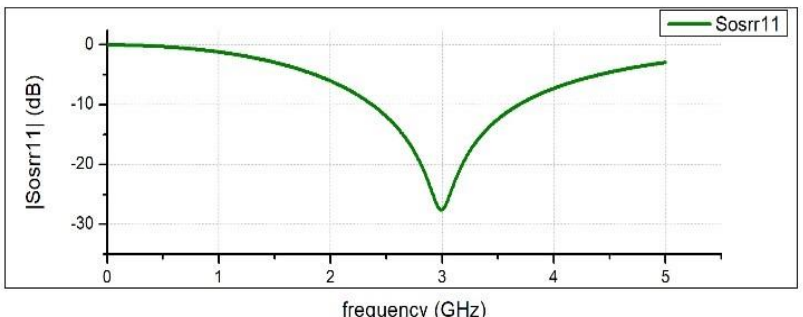

(a)

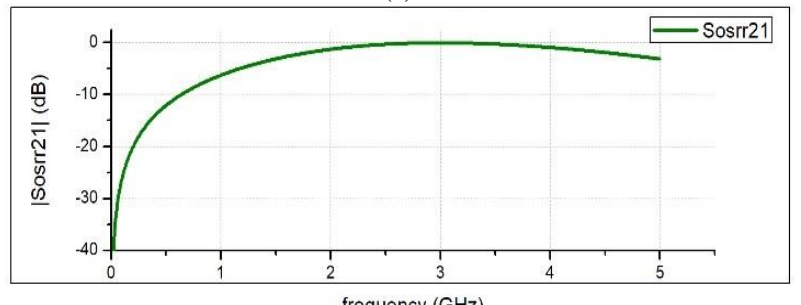

(b)

Fig. 8. Representation of Scattering Parameter of OSRR (a) S SSRR11 (Reflection Coefficient) (b) S SOSRR21 (Transmission Coefficient).

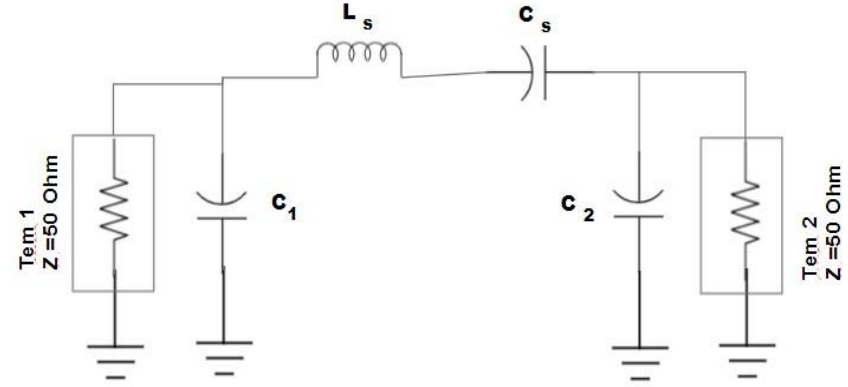

Fig. 9. Equivalent Circuit of OSRR.

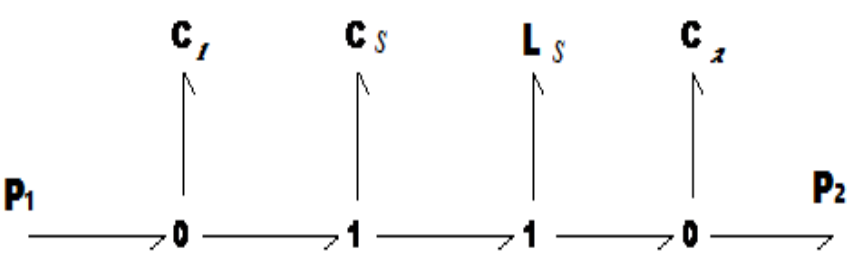

Fig. 10. Acausal Bond Graph Model of OSRR

By applying the causal assignment rules and the equivalent schema reduction properties of the bond graph approach, we obtain the simplified model shown in Fig. 1.

From the Bond Graph model [12], we can deduce that the model is of flow-flow causality, this structure is represented as a parallel branch (0-junction) by a capacitance $\mathrm{C}_{1}$ (admittance $\mathrm{Y}_{\mathrm{c} 1}$ ), in series (1-junction) by an inductance $\mathrm{L}_{\mathrm{S}}$ and a capacitance $\mathrm{C}_{\mathrm{S}}$ (Impedance $\mathrm{Z}_{\text {éq }}$ ), in parallel (0-junction) by a capacitance $\mathrm{C}_{2}$ (admittance $\mathrm{Y}_{\mathrm{c} 2}$ ) [15]. This allows us to write the following expressions (normalization makes it easy to calculate later):

$Y_{C_{1}}=\stackrel{\leftarrow}{\longrightarrow} \frac{1}{j \omega C_{1}} \quad y_{c_{1}}=\tau_{C_{1}} S$

(2)

$Z_{\text {é } q}=\frac{1}{j \omega C_{S}}+j \omega L_{s} \quad Z_{\text {é } q}=\left(\frac{1}{\tau_{c_{S}}}+\tau_{L_{S}}\right) s$

$Y_{C_{2}}=\frac{1}{j \omega C_{2}} \quad y_{c_{2}}=\tau_{C_{2}} S$

With s : Laplace operator and $\tau_{x}$ normalized constant.

As shown in Fig. 11, the OSRR consists of three submodels respectively parallel-serial-parallel, and the determination of the wave matrix $\left[W_{(O S R R)}\right]$ from the model representation Bond Graph.

$$
\begin{aligned}
& {\left[W_{(\text {OSRR })}\right]=\left(\begin{array}{ll}
W_{(\text {OSRR })_{11}} & W_{(\text {OSRR })_{12}} \\
W_{(\text {OSRR })_{21}} & W_{(\text {OSRR })_{22}}
\end{array}\right)} \\
& W_{(\text {OSRR })_{11}}=\frac{\left[z_{1}\left(y_{c_{1}} y_{c_{2}}+y_{c_{1}}+y_{c_{2}}+1\right)+y_{c_{1}}+y_{c_{2}}+2\right]}{2} \\
& W_{(\text {OSRR })_{12}}=\frac{\left[z_{1}\left(y_{c_{1}} y_{c_{2}}-y_{c_{1}}+y_{c_{2}}-1\right)+y_{c_{1}}+y_{c_{2}}\right]}{2} \\
& W_{(\text {OSRR })_{21}}=\frac{\left[z_{1}\left(y_{c_{1}} y_{c_{2}}-y_{c_{1}}+y_{c_{2}}-1\right)-y_{c_{1}}-y_{c_{2}}\right]}{2} \\
& W_{(\text {OSRR })_{22}}=\frac{\left[z_{1}\left(-y_{c_{1}} y_{c_{2}}+y_{c_{1}}+y_{c_{2}}-1\right)-y_{c_{1}}-y_{c_{2}}+2\right]}{2}
\end{aligned}
$$

The matrix $\left[W_{(O S R R)}\right]$ allows us to find the scattering matrix $\left[S_{(O S R R)}\right]$ through the relation (1) (the parameters are represented taking into account that the values of the 
capacitances $\mathrm{C}_{1}$ and $\mathrm{C}_{2}$ are equal, so we have to replace $\mathrm{y}_{\mathrm{c} 1}$ and $\mathrm{y}_{\mathrm{c} 2}$ by $\left.\mathrm{y}_{\mathrm{osrr}}\right)$.

$S_{(O S R R)_{11}}=\frac{1}{2} \frac{y_{o s r r}^{2} z_{e q}+2 y_{o s r r} z_{e ́}+2 y_{o s r r}+z_{e q}+2}{-y_{o s r r}^{2} z_{e q}+2 y_{o s r r} z_{e q}-2 y_{o s r r}-z_{e q}+2}$

$S_{(O S R R)_{12}}=\frac{2}{-y_{\text {osrr }}^{2} z_{\text {é } q}+2 y_{\text {osrr }} z_{\text {é } q}-2 y_{o s r r}-z_{\text {é }}+2}$

$S_{(O S R R)_{21}}=\frac{2}{-y_{\text {osrr }}^{2} z_{\text {éq }}+2 y_{o s r r} z_{\text {é }}-2 y_{o s r r}{ }^{-z_{\text {é }} q^{+2}}}$

$S_{(O S R R)_{22}}=\frac{1}{2} \frac{-\left(y_{\text {osrr }}^{2} z_{\text {é } q}-z_{\text {é } q}+2 y_{o s r r}\right)}{-y_{\text {osrr }}^{2} z_{\text {éq }}+2 y_{o s r r} z_{\text {é } q}-2 y_{o s r r}-z_{\text {é } q}+2}$

The parameters of the lumped elements used for the simulation OSRR and obtained in Fig. 12 are presented in Table I.

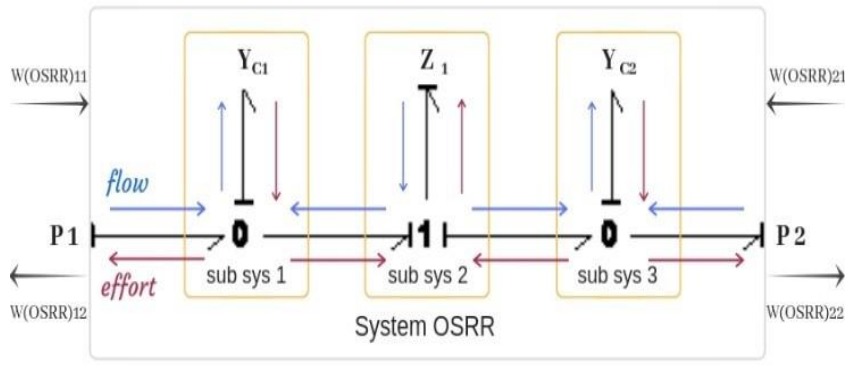

Fig. 11. Reducted and Causal Bond Graph Model of OSRR.

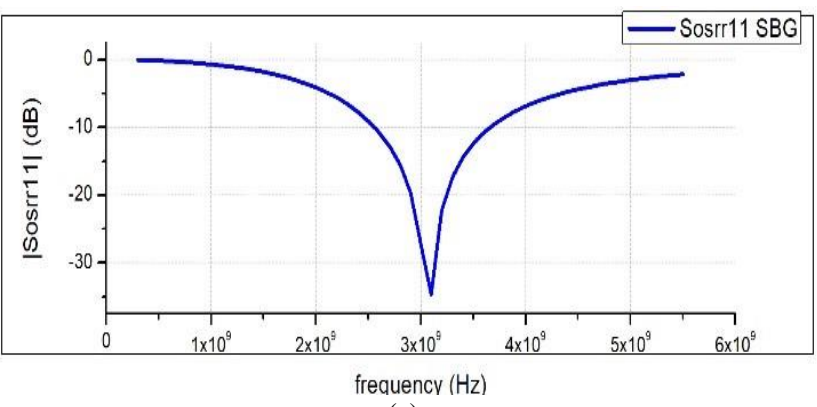

(a)

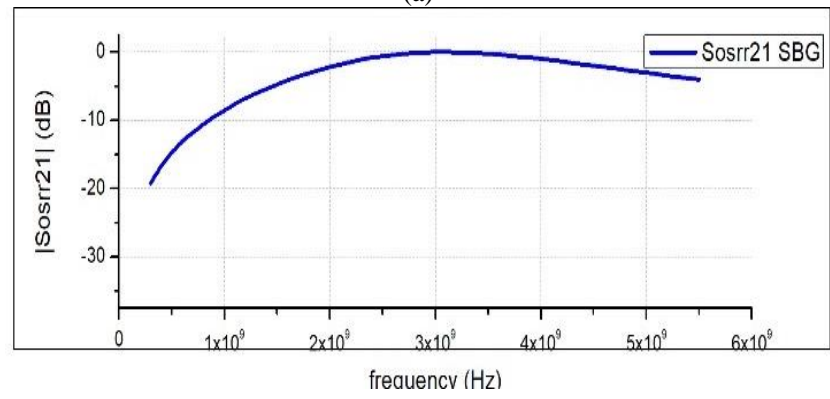

(b)

Fig. 12. Simulation Result of OSRR Equivalent Bond Graph Model (a) S OCSRR11 (Reflection Coefficient) (b) S S OCSRR21 (Transmission Coefficient).

TABLE I. VALUES OF THE ELEMENTS OF THE PROPOSED OSRR CirCUIT

\begin{tabular}{|l|l|l|l|l|}
\hline Element & $\mathbf{C}_{\mathbf{1}}$ & $\mathbf{C}_{\mathbf{s}}$ & $\mathbf{L}_{\mathbf{s}}$ & $\mathbf{C}_{\mathbf{2}}$ \\
\hline Value & $10 \mathrm{pF}$ & $5.55 \mathrm{nH}$ & $0.58 \mathrm{pF}$ & $\mathrm{F}$ \\
\hline
\end{tabular}

\section{B. Synthesis of OCSRR (Complementary Open Split-Ring Resonator)}

In 2009, Vélez et al [16] were able to present for the first time the OCSRR, which obtained it the application of the duality (we can also say that it is his complementary image) of OSRR.

The OCSRR structure can be realized by a pair of two rings which are concentrically placed with slots placed in the same position where this pair is placed symmetrically. And the role of the extra band back of the design and vias is to connect the ground plane.

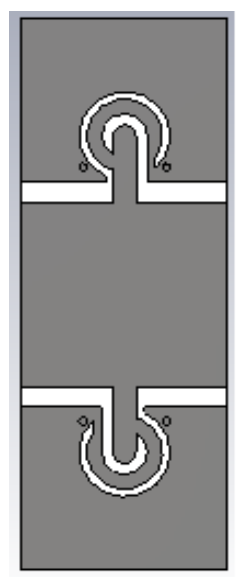

(a)

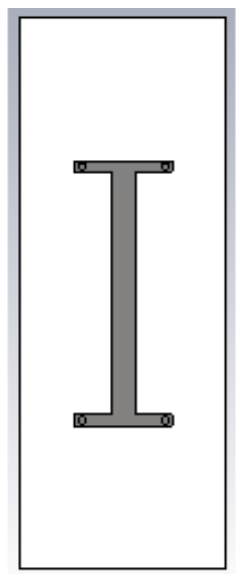

(b)
Fig. 13. Topologie of a Series Connected OCSRR in a CPW Transmission Line (a) Front view (b) Rear View.

The configuration of the OCSRR is illustrated in Fig. 13 where the substrate considered is the Rogers RO3010 with a thickness $h_{o c r r}=0,254 \mathrm{~mm}$ and a dielectric constant $\varepsilon_{\mathrm{r}}=11,2$.

The simulation of the scattering parameters [S] of the Topology of a series connected OCSRR in a CPW transmission line gives the results presented in Fig. 14.
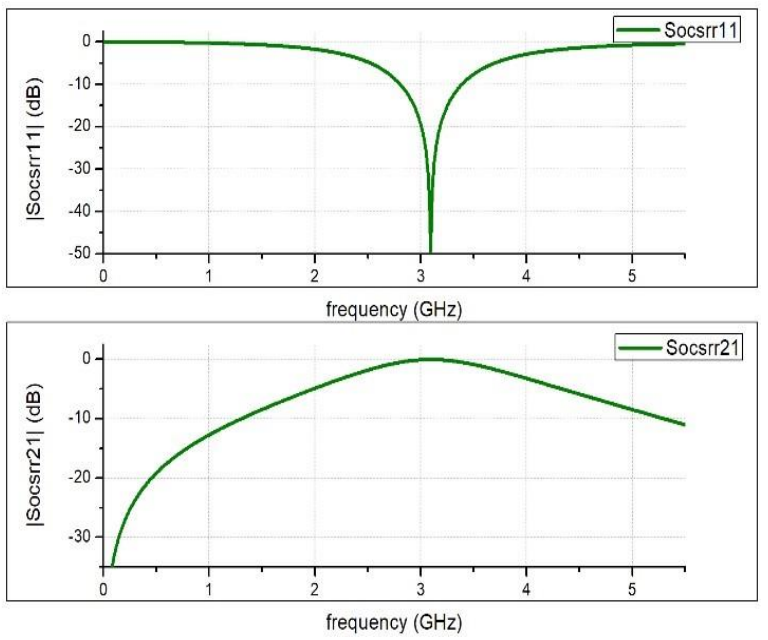

Fig. 14. Representation of Scattering Parameter of OCSRR (a) S SCSRR11 (Reflection Coefficient)(b)S SocsRR21 (Transmission Coefficient).

The proposal of the resonator equivalent circuit OCSRR can be represented by a $\mathrm{T}$ circuit (presented by localized 
elements $C_{p}, L_{p}, L_{1}$ and $L_{2}$ ) as shown in Fig. 15 with a source and load equal to $50 \mathrm{ohms}$.

The acausal Bond Graph model of a resonator of the OSRR type can be represented by the Fig. 16 [11] [12].

By applying the causal assignment rules and the equivalent schema reduction properties of the bond graph approach, we obtain the simplified model shown in Fig. 17.

From the Bond Graph model [17], we can deduce that the model is of effort-effort causality, this structure is represented as a series branch (1-junction) by an inductance $\mathrm{L}_{1}$ (Impedance $\mathrm{Z}_{\mathrm{L} 1}$ ), in parallel (0-junction) by an inductance $\mathrm{L}_{\mathrm{p}}$ and a capacitance $\mathrm{C}_{\mathrm{p}}$ (Admittance $\mathrm{Y}_{\mathrm{eq}}$ ), in series (1-junction) by an inductance $\mathrm{L}_{2}$ (Impedance $\mathrm{Z}_{\mathrm{L} 2}$ ). This allows us to write the following expressions (normalization makes it easy to calculate later):

$Z_{L_{1}}=\frac{1}{j \omega L_{1}} \longleftrightarrow Z_{L_{1}}=\tau_{L_{1}} S$

$Y_{e q}=\frac{1}{j \omega C_{p}}+j \omega L_{p} \quad y_{e q}=\left(\frac{1}{\tau_{L p}}+\tau_{c_{p}}\right) s$

$Z_{L_{2}}=\frac{1}{j \omega L_{2}} \quad Z_{L_{2}}=\tau_{L_{2}} S$

As shown in Fig. 17, the OCSRR consists of three submodels respectively series-parallel-series, and the determination of the wave matrix $\left[W_{(\text {OCSRR })}\right]$ from the model representation Bond Graph.

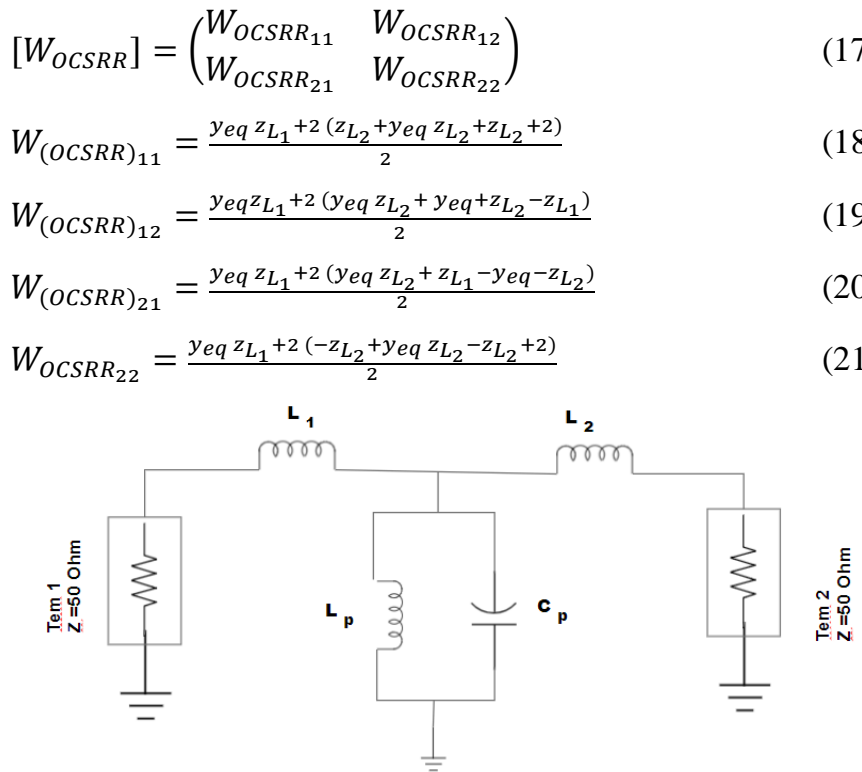

Fig. 15. Equivalent Circuit of OCSRR.

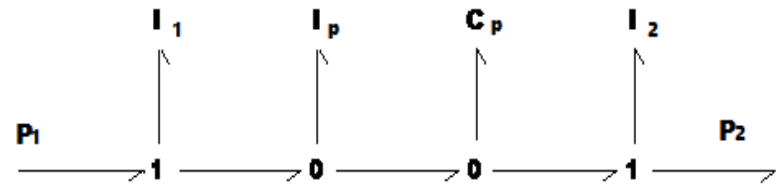

Fig. 16. Acausal Bond Graph Model of OCSRR.

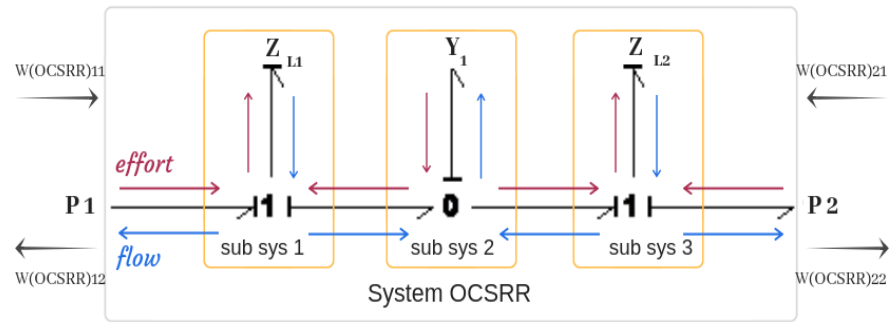

Fig. 17. Reducted and Causal Bond Graph Model of OCSRR.

The matrix $\left[W_{(O C S R R)}\right]$ allows us to find the scattering matrix $\left[S_{(O C S R R)}\right]$ through the relation (1) (the parameters are represented taking into account that the values of the inductance $L_{1}$ and $L_{2}$ are equal, so we have to replace $Z_{L 1}$ and $\mathrm{Z}_{\mathrm{L} 2}$ by $\left.\mathrm{Z}_{\mathrm{ocsrr}}\right)$.

$S_{(O C S R R)_{11}}=\frac{y_{e q} z_{o c s r r}^{2}+2 y_{e q} z_{o c s r r}+2 z_{o c s r r}+y_{e q}+2}{-y_{e q} z_{o c s r r}^{2}+2 y_{e q} z_{o c s r r}-2 z_{o c s r r}-y_{e q}+2}$

$S_{(O C S R R)_{12}}=\frac{2}{-y_{e q} z_{o c s r r}^{2}+2 y_{e q} z_{o c s r r}-2 z_{o c s r r}-y_{e q}+2}$

$S_{(\text {OCSRR })_{21}}=\frac{2}{-y_{e q} z_{o c s r r}^{2}+2 y_{e q} z_{o c s r r}-2 z_{o c s r r}-y_{e q}+2}$

$S_{(\text {OCSRR })_{22}}=\frac{-y_{e q} z_{\text {ocsrr }}^{2}+y_{e q}-2 z_{o c s r r}}{-y_{e q} z_{o c s r r}^{2}+2 y_{e q} z_{o c s r r}-2 z_{o c s r r}-y_{e q}+2}$

The parameters of the lumped elements used for the simulation OCSRR and obtained in Fig. 18 are presented in Table II.
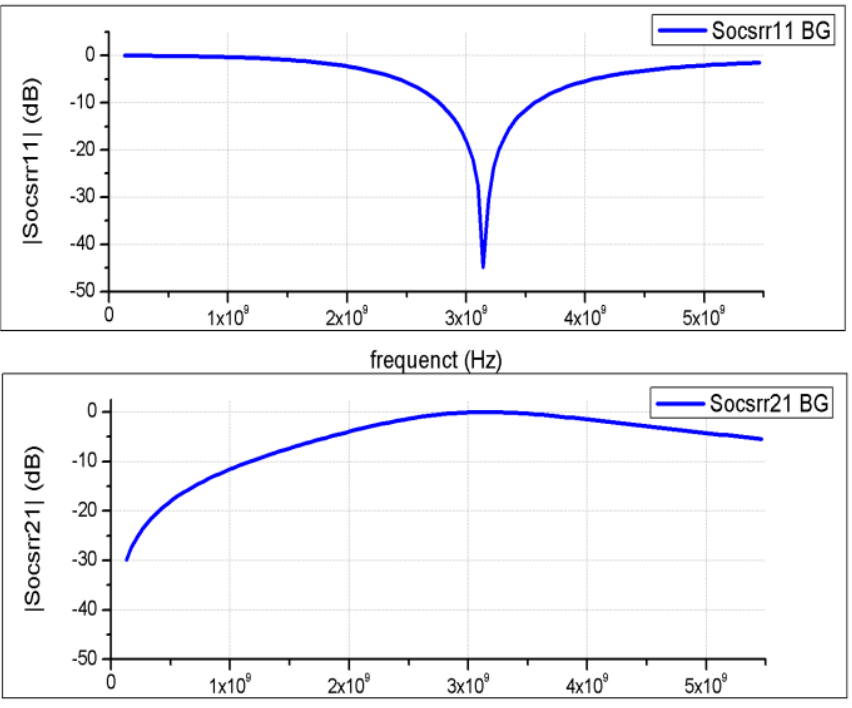

Frequency $(\mathrm{Hz})$

Fig. 18. Simulation Result of OCSRR Equivalent Bond Graph Model (a) SosCRR11 (Reflection Coefficient) (b) SOCSRR21 (Transmission Coefficient).

TABLE II. VALUES OF THE ELEMENTS OF THE PROPOSED OSCRR CIRCUIT

\begin{tabular}{|l|l|l|l|l|}
\hline Element & $\mathbf{L}_{\mathbf{1}}$ & $\mathbf{L}_{\mathbf{p}}$ & $\mathbf{C}_{\mathbf{p}}$ & $\mathbf{L}_{\mathbf{2}}$ \\
\hline Value & $0.32 \mathrm{nH}$ & $0.983 \mathrm{nH}$ & $2.85 \mathrm{pF}$ & $0.32 \mathrm{H}$ \\
\hline
\end{tabular}




\section{RESULT AND DISCUSSION}

In this section, a comparison between the results of the electromagnetic simulation and the simulation with the Scattering Bond Graph methodology of the OSRR and OCSRR resonators.

\section{A. OSRR (Open Spilt-Ring Resonator)}

Fig. 19 shows a comparison of the variation of reflection coefficient and the variation of transmission coefficient as a function of frequency variation. It's noted that the resonance frequency (the frequency where the OSRR is better adapted) $F_{\text {res }(\mathrm{OSRR})}=\mathrm{F}_{\text {res }}(\mathrm{OSRR}) \mathrm{BG}=3.1 \mathrm{GHz}$, and that the loss (the difference between the power of an input and output of a cavity at the resonant frequency) is zero, which confirms the total transmission of the waves incident.

The return losses are greater than $-15 \mathrm{~dB}$ at the resonant frequency and the bandwidth width $\Delta \mathrm{f}_{(\mathrm{OSRR})}=0,7 \mathrm{MHz}$ and $\Delta \mathrm{f}_{(\mathrm{OSRR}) \mathrm{BG}}=0,57 \mathrm{MHz}$. With the bandwidth $\mathrm{BP}$ (OSRR) $=$ $22,83 \%$ and $\mathrm{BP}{ }_{(\mathrm{OSRR}) \mathrm{BG}}=17,6 \%$.

The characterization of the frequency response by the two techniques (electromagnetic and Scattering Bond Graph) shows good agreement with some slight differences.

\section{B. OCSRR (Complementary Open Split-Ring Resonator)}

It's remarkable in Fig. 20 that the minimum value of the reflection coefficient (SOCSRR11 BG and SOCSRR11) which corresponds to the frequency of resonance $F_{\text {res }}$ (OCSRR) $=$ $\mathrm{F}_{\text {res(OCSRR) BG }}=3,1 \mathrm{GHz}$.

The return losses are greater than $-15 \mathrm{~dB}$ at the resonant frequency and the bandwidth width $\Delta \mathrm{f}_{(\mathrm{OCSRR})}=0,3 \mathrm{MHz}$ and $\Delta \mathrm{f}_{(\mathrm{OCSRR}) \mathrm{BG}}=0,4 \mathrm{MHz}$. With the bandwidth $\mathrm{BP}{ }_{(\mathrm{OCSRR})}=$ $10,2 \%$ and $\mathrm{BP}$ (OSRR)BG $=12,4 \%$.

It is important to highlight the importance and effectiveness of the use of the Scattering Bond Graph approach, which allows us to extract frequent parameters with a very satisfying precision.

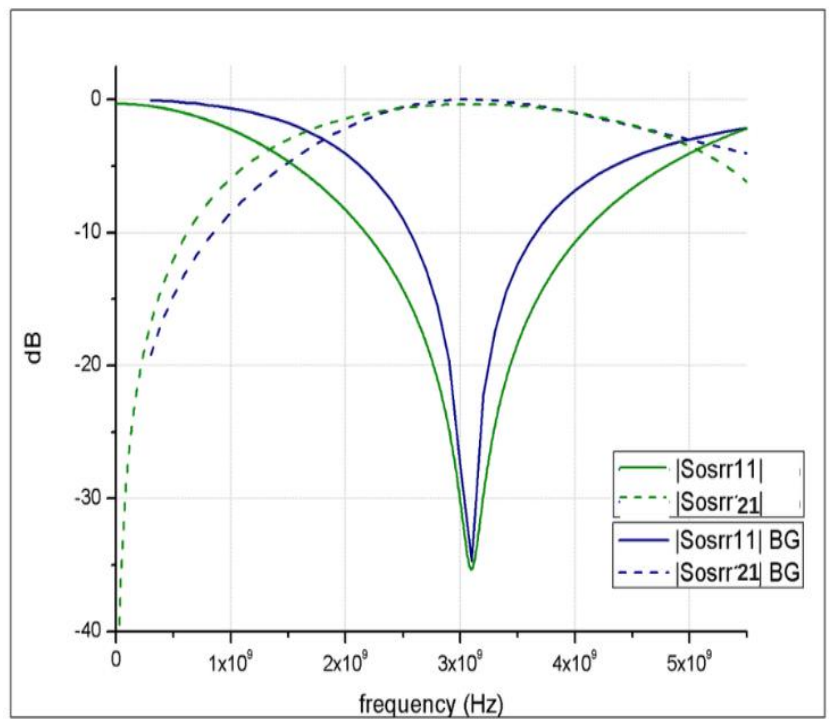

Fig. 19. Frequency Response Comparison of the OSRR between the Electromagnetic Method and SBG Methodology.

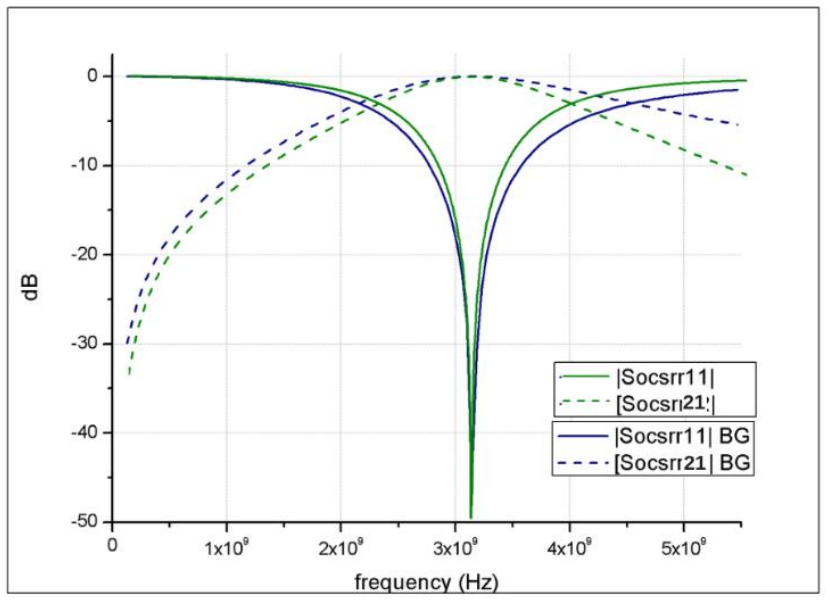

Fig. 20. Frequency Reponse Comparaison of the OCSRR between the Electromagnetic Method and SBG Methodology.

\section{CONCLUSION}

The objective of this work is to prove the contribution and the modelling of the new study methodology called dispersion link graph, in the analysis of transmission lines of metamaterials loaded with OSRR resonators "Open Split-Ring Resonators" and COSRR "Complementary Open Split-Ring Resonators" of equivalent electrical diagrams. In a first part, we studied the electromagnetic responses resulting from the topology proposed by each resonator, then we gave the modelling by the Bond Graph approach of the proposed equivalent circuit. In a second part, we proved by simulation that the frequency responses can be deduced by a simple matrix relation as a function of the wave matrix [W] (matrix extracted from the modelling diagram by Bond Graph (BG) on the based on the characteristic properties of the SBG methodology). In the third part, we validated our results by comparing the results between SBG and the electromagnetic methodology.

The particular properties of the Scattering Bond Graph technique allowed us to easily deduce the wave matrix [W], whatever the complexity of the system which facilitates the deduction of the diffusion matrix [S], which allows us to find and analyze the frequency parameters without problem of adaptation between the sub-models. In our case we have modeling and Analyses the Equivalent-Schema Models for Open Split-Ring Resonators and Complementary Open SplitRing Resonators Coupled to Planar Transmission Lines. Thus, we were able to validate our results by this comparison which seems to be an excellent means to follow in the field of radiofrequency characterization.

In future work we will take into account nonlinearities in the physical system of the matrix scattering type depending on the entered vector, by applying the concept of generalized impedance.

\section{REFERENCES}

[1] R. S. Daniel, R. Pandeeswari, and S. Raghavan, "Design and Analysis of Open Complementary Split Ring Resonators Loaded Monopole Antenna for Multiband Operation," vol. 78, no. July, pp. 173-182, 2017.

[2] V. G. Veselago, "The Electrodynamics of Substances with Simultaneously Negative Values of and $\mu$," vol. 509, 1968. 
[3] Tie Jun Cui • David R. Smith • Ruopeng Liu Editors, Ed., Métamatrials, Theory, Design and Application, Springer. .

[4] R. A. Shelby, D. R. Smith, and S. Schultz, "Experimental Verification of a Negative Index of Refraction," vol. 292, no. April, 2001.

[5] C. Effects, J. Naqui, S. Member, M. Durán-sindreu, and F. Martín, "Modeling Split-Ring Resonator ( SRR ) and Complementary Split-Ring Resonator ( CSRR ) Loaded Transmission Lines Exhibiting," vol. 12, pp. 178-181, 2013.

[6] A. Vélez et al., "Open Complementary Split Ring Resonators ( OCSRRs ) and Their Application to Wideband CPW Band Pass Filters," vol. 19, no. 4, pp. 197-199, 2009.

[7] N. Kou, Y. Shi, and L. Li, "New equivalent circuit analysis and synthesis for broadband composite right/left-handed transmission line Metamaterials," Appl. Comput. Electromagn. Soc. J., vol. 31, no. 8, pp. 884-893, 2016.

[8] H. Paynter and M. M. I. T. Press, "Analysis and design of engineering systems : class notes for M I T .course 2. 751 / by Henry M . Paynter; with the assistance of Peter Briggs .not subject to copyright. Users are free to copy , use ," 1961.

[9] K. Kurokwa, "Power Waves," no. 1, pp. 194-202, 1964.

[10] H. Taghouti, A. Mami, and B. Graph, "Extraction , Modelling and Simulation of the Scattering Matrix of a Chebychev Low-Pass Filter with cut-off frequency $100 \mathrm{MHz}$ from its Causal and Decomposed Bond Graph Model,”vol. 10, no. 1, pp. 29-37, 2010.
[11] H. Taghouti, A. Mami, and T. Le Belvedere, "Discussion around the Scattering Matrix Realization of a Microwave Filter using the Bond Graph Approach and Scattering Formalism Department of Electrical Engineering, Laboratory of Analysis and Command of the Systems, Department of Physics, Faculty of ," vol. 9, no. 4, pp. 459-467, 2012.

[12] I. Salem, H. Taghouti, and A. Mami, "Modeling CSRR and OCSRR loaded transmission line by bond graph approach methodology," 2018 9th Int. Renew. Energy Congr. IREC 2018, no. Irec, pp. 1-6, 2018.

[13] F. Falcone et al., "Effective Negative- " Stopband Microstrip Lines Based on Complementary Split Ring Resonators," vol. 14, no. 6, pp. 280-282, 2004.

[14] J. Naqui, F. Paredes, and J. Bonache, "Electrically Small Resonators for Planar Metamaterial, Microwave Circuit and Antenna Design: A Comparative Analysis," no. December, 2012.

[15] J. Sabri, N. Omrane, T. Hichem, M. Abdelkader, and G. Ali, "Miniaturized Meander Slot Antenna Tor RFID TAG with Dielectric Resonator at 60 Ghz," Int. J. Adv. Comput. Sci. Appl., vol. 7, no. 4, pp. 373-380, 2016.

[16] F. Aznar-ballesta, M. Gil, and M. Durán-sindreu, "Characterization of Metamaterial Transmission Lines with Coupled Resonators Through Parameter Extraction," 2009.

[17] I. Salem, H. Taghouti, A. Rahmani, and A. Mami, "Application of the Scattering Bond Graph Methodology for Composite Right / Left Handed Transmission Lines," vol. 10, no. 6, pp. 19-24, 2019. 